\title{
A new remote sensing filter radiometer employing a Fabry-Perot etalon and a CCD camera for column measurements of methane in the Earth atmosphere
}

\author{
Georgieva, E.M ${ }^{1}$, Huang W. ${ }^{3}$, Heaps, W.S. ${ }^{2}$ \\ ${ }^{1}$ Joint Center for Earth Systems Technology (JCET), UMBC, Baltimore, MD 21228, USA \\ e-mail: Elena.M.Georgieva@nasa.gov \\ ${ }^{2}$ NASA, Goddard Space flight Center, Greenbelt, MD 20771, USA \\ ${ }^{3}$ Science Systems and Applications, Inc., Lanham, MD, USAA
}

\begin{abstract}
A portable remote sensing system for precision column measurements of methane has been developed, built and tested at NASA GSFC. The sensor covers the spectral range from $1.636 \mu \mathrm{m}$ to $1.646 \mu \mathrm{m}$, employs an air-gapped Fabry-Perot filter and a CCD camera and has a potential to operate from a variety of platforms. The detector is an XS-1.7-320 camera unit from Xenics Infrared solutions ${ }^{1}$ which combines an uncooled InGaAs detector array working up to $1.7 \mu \mathrm{m}$. Custom software was developed in addition to the graphical user basic interface $\mathrm{X}$-Control provided by the company to help save and process the data. The technique and setup can be used to measure other trace gases in the atmosphere with minimal changes of the etalon and the prefilter. In this paper we describe the calibration of the system using several different approaches.
\end{abstract}

Instrumentation; measurement; metrology; remote sensing; atmospheric composition; optical instruments; absorption; interferometry; Fabry-Perot.

\section{INTRODUCTION}

Methane measurements are becoming increasingly important as a component of NASA's programs to understand the global carbon cycle and quantify the threat of global warming. Methane is the third most important greenhouse gas in the Earth's radiation budget (after water vapor and carbon dioxide) and the second most important anthropogenic contributor to global warming. Individual molecules of methane are 25 more effective than carbon dioxide in trapping heat in the earth's atmosphere. In its 2007 report the International Panel on Climate Change identified methane as a key uncertainty in our understanding saying that "the causes of recent changes in the growth rate of atmospheric $\mathrm{CH}_{4}$ are not well understood" ${ }^{2}$. In US global change research program strategic plan 2012-202 is stated that "The primary cause of climate change is increasing greenhouse gases in the atmosphere, mainly carbon dioxide and methane." We do know that methane arises from a number of natural sources including wet lands and the oceans plus man made sources from agriculture, as well as coal and petroleum production and distribution. It has recently been pointed out that large amounts of methane are frozen in the permafrost of Canada and Siberia. There is a fear that melting of this permafrost driven by global warming may release methane very suddenly further exacerbating climate change $\mathrm{e}^{3-6}$.

\section{DESCRIPTION OF INSTRUMENT AND BASIC THEORY}

In the current setup (Fig.1) sunlight enters the instrument from the right, passes through a bandpass filter and is then split. One half goes directly to a detector and the other half pass through a Fabry-Perot interferometer ${ }^{7,8}$ tuned to transmit at wavelengths where methane absorbs light. The detector for this half then responds strongly to changes in the atmospheric methane. The difference with the previous setup reported ${ }^{10-14}$ is that instead of two InGaAs detectors for the Reference and FP channel we use a CCD array to sample both the reference and the Fabry-Perot signal simultaneously on different areas of the array. It increases the signal to noise ratio ${ }^{9}$ (SNR) while permitting a reduction in the overall size of the instrument.

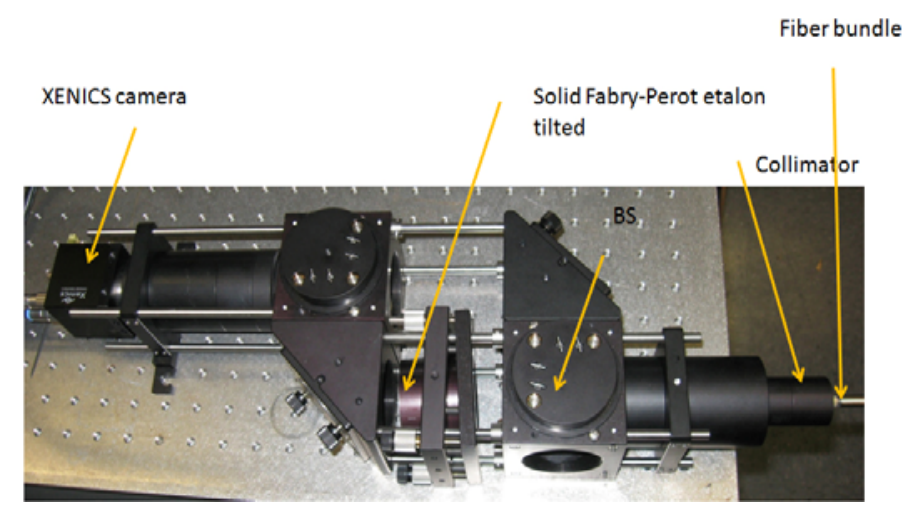

Figure 1 shows the sesor with the Xenics CCD camera.

\section{EXPERIMENTAL}

As with any instrument, calibration and validation are crucial to the success of the measurement. To effectively address questions regarding sources and sinks of methane, precision at the level of $1.0 \%$ or better is required. 
The methane filter radiometer instrument uses a differential optical absorption measurement technique. In this technique, it makes measurements of light at an atmospheric absorption wavelength and in a nearby atmospheric transmission wavelength region. The process for converting these measurements to a mixing ratio determination for an optical

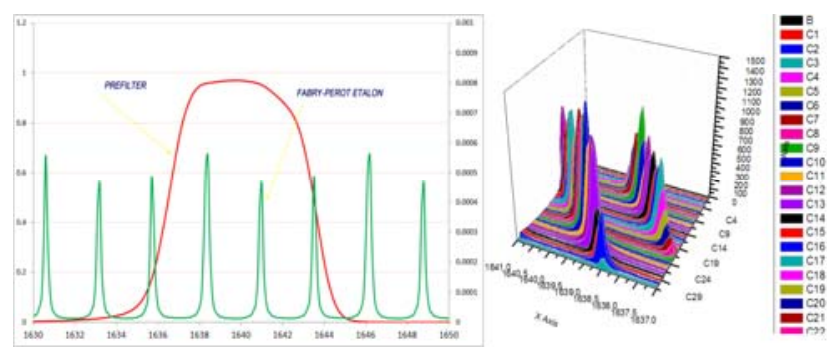

Figure 2, Left-FP transmission bands and prefilter shape Right: shows the transmitted wavelengths on the CCD array

species involves a ratio of these two measurements, which were obtained using very nearly the same instrumental components. Because of this, a change in the reflectivity of a mirror or the transmission of some component in the common optical path "divides out" so such changes do not affect the derived mixing ratio.

The methane instrument calibration/validation covers three areas-spectral, radiometric, and pointing. The spectral calibration proceeds from ground testing.

\section{A. Wavelength calibration}

The radiometer has been calibrated by different methods using sunlight, an Agilent tunable laser and using calibrated lamps and integrating spheres in the Radiometric facility at Goddard. For the first method because of the difficulty in testing the camera from the roof directly pointing it at the sun we implemented a fiber optically coupled system in which a roof mounted suntracker produces an image of the sun on a fiber bundle. The fiber bundle is led down into the

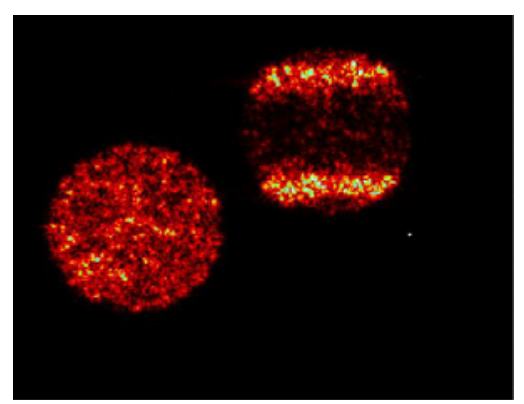

Figure 3, Images of Ref and FP channel during the wavelength calibration third floor laboratory and the light is introduced into the Xenics camera. Two Lab View software programs were developed to assist in wavelength camera calibration and the analysis of the collected data. Tunable laser light from a source locked to a spectral reference line is introduced to the sensor via multimode fiber coupled to a fiber bundle. Tuning the laser permits a wavelength map to be made of the "bulls-eye" pattern in the focal plane (Fig.3)

We first use a tunable laser (Agilent 81649) and a single mode fiber and look at the displacement of FP and Reference channel images on the CCD array. In this way the images become minimal in size. We adjust the vertical and horizontal positions of the images so they look similar for both the FP and Reference channels. The displacement can be obtained from the image data with an error $<0.5$ pixel. Than we use a fiber bundle to input a uniformly illuminated Agilent laser beam into the sensor. The laser wavelength was changed in steps of $0.1 \mathrm{~nm}$ from $1630 \mathrm{~nm}$ until $1641 \mathrm{~nm}$, which is the maximum wavelength for our laser.

We record images for several wavelengths to derive the free spectrum range of the FP and extrapolate the fringe position for wavelengths from $1641 \mathrm{~nm}$ to $1650 \mathrm{~nm}$. The Agilent laser wavelength can be calibrated using $\mathrm{CH}_{4}$ lines below $1641 \mathrm{~nm}$.

Figure 4 shows a screenshot taken November 28, 2011 Each wavelength is a line in the curves on the right. The two images on the two-dimensional IR detector array vary in wavelength along the array diagonal. The left image is our Reference channel and the right image is the Fabry-Perot channel. The comb filter that we use is an air-gapped etalon with diameter $50.8 \mathrm{~mm}, 80 \%$ reflection at 1565-1670 nm and free spectral range (FSR) of $2.56 \mathrm{~nm}$. The etalon is tuned by tilting at $10 \mathrm{deg}$. Intensity variation is a result of passing clouds. Each point in the curve is $1 \mathrm{~ms}$ integration of CCD. Shown are the focal plane images of a hexagonal fiber optic array feeding the methane sensor from a roof mounted suntracker (Fig5). The right hexagon is the

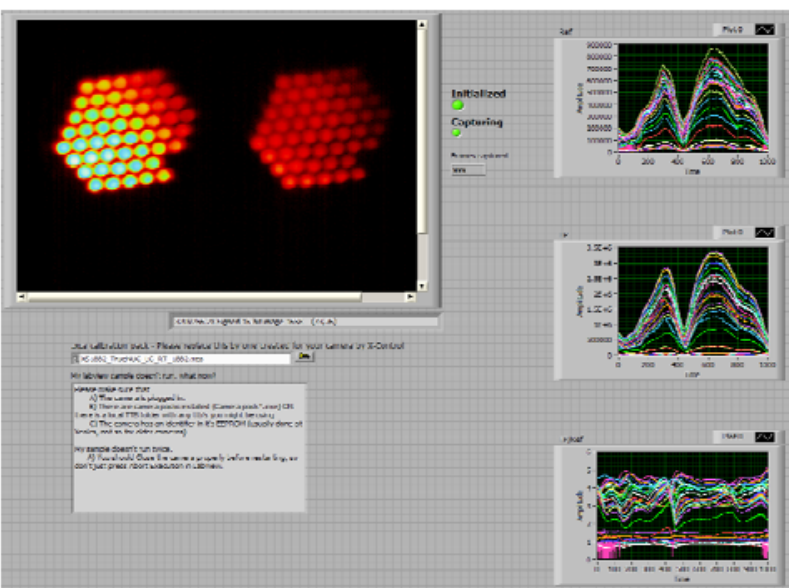

Figure 4 A screenshot with sunimages and panels for Reference, FP and Ratio channels

reference channel and the left hexagon has been encoded in wavelength by passing through a Fabry-Perot solid etalon before imaging. The (almost) horizontal band of pink across the left hexagon is caused by methane absorption at the wavelengths represented by those pixels. The color change at the right edge of both hexagons is caused by cloud partly obscuring the solar image. 


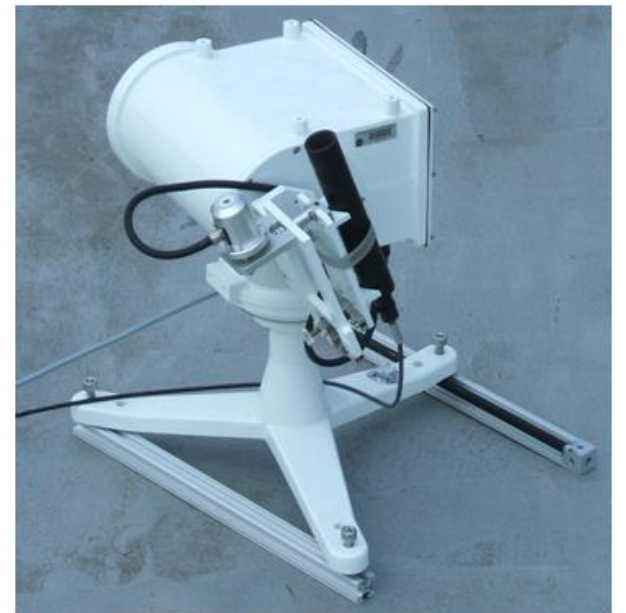

Figure 5. The telescope attached to the suntracker focuses an image of the sun on the end of a 52 fiber bundle used to conduct light down to the instrument in the lab.

After normalizing the left image by the right one the intensity of each dot can be plotted against the wavelength that it represents to produce a short absorption spectrum for methane.

For the last calibration method we used bulbs in various combinations and an integrating sphere, so a range of output illuminations can be achieved.

\section{B. Intensity calibration}

The camera had to be calibrated against a known radiation source. It converts the arbitrary units of the camera into physical units of energy. Here we discuss the radiometry theory required to calibrate the camera and apply this calibration to field images. Radiometric calibration is

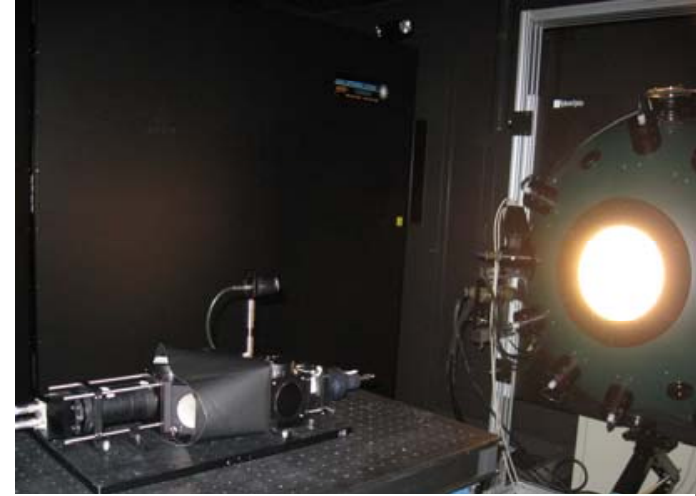

Figure 6 Grande $1 \mathrm{~m}$ diameter sphere with the sensor in front of it, Goddard Calibration Facility

accomplished using a NIST-calibrated white light source; a pixel map is generated for the focal plane array. This includes sensitivity, dark current, and read noise. A full instrument calibration again generates a pixel map that is wavelength-specific the array.

Integrating sphere systems are highly Lambertian and show very uniform radiance distribution. We measured the linearity of the camera by irradiating the array with varying light levels and measuring the signal produced.

\section{Preliminary Data collection using a fibercoupled suntracker}

Light for the measurement is gathered by a sun tracker on the roof that focuses an image of the sun onto the end of a fiber bundle. Each fiber within the bundle collects light from a different place on the sun and the instrument forms an image of each fiber in the bundle. Images of the fiber bundle FP and REF channel have been shown in Figure 4. Figure 7 shows results from roughly three days of continuous observation by the suntracker. The traces labeled "C" correspond to different rings in the Fabry-Perot's mapping the pixels. Zero time begins on the afternoon of March 8, 2012. Rings C15 through C18 show an initial decrease. This is because as the sun sets the atmospheric path length grows longer and the effect of methane absorption grows stronger.

Rings C14 and C19 are not mapped onto a wavelength that methane absorbs so they do not change. Seconds 1000 through 5500 represent sunset, night when the FP and REF signals are near zero, and then sunrise. During sunrise and sunset the pathlength becomes very long and the probability of clouds in the path becomes very high. This is why the ratio becomes extremely noisy during these transitions.

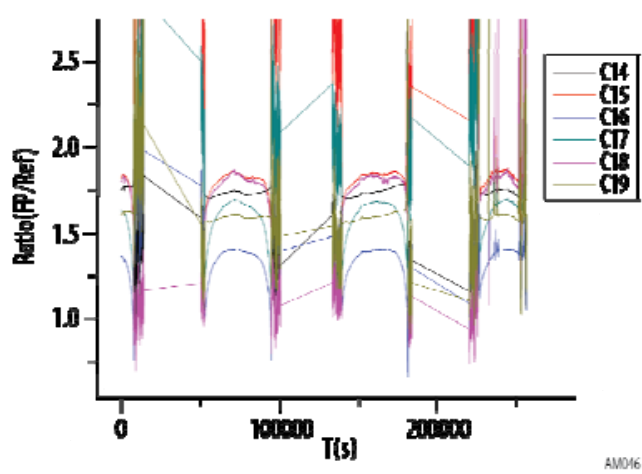

Figure 7. This shows the FP/REF ratio plotted for almost four days of continuous operation. Only data from the day time periods has value.

During the first full day (March 9) rings C15 - C 18 show in increase during the morning as the atmospheric path length grows shorter reaching a minimum at local noon and then increasing again in the afternoon as on March 8. Rings C14 and C19 show little variation because they are off the methane absorption. Two additional nights and days are 
shown exhibiting the same pattern. March 11 is very noisy because the day was mostly cloudy.

Figure 8 shows data taken at two times in March 9 - early morning and noon. Rows 11, 12, 13, and 14 corresponding to rings C15, C16, C17, and C18 in Figure 7 show a variation in ratio caused by changes in the airmass between these two times of day. Other rings less sensitive to methane show no such variation. We can calculate the pathlength from the instrument to the sun very precisely.

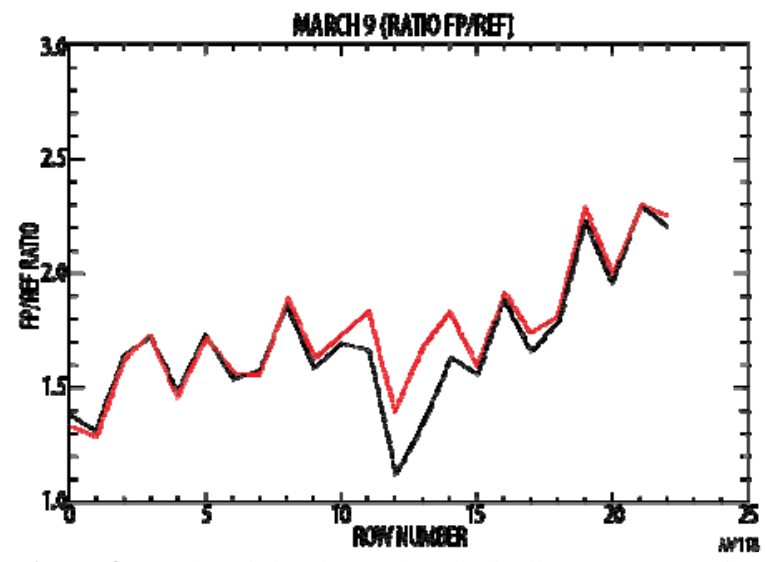

Figure 8. A plot of the ring ratios (basically a spectrum) for two times of day. Row 12 (ring C17) shows a large change between the deep trough for the early morning data and the shallower trough for noon time data. Rows that do not lie on the methane absorption line do not show any variation.

Knowing the wavelength assignments of the pixels and the methane absorption spectrum will permit an accurate determination of the column methane concentration. The final wavelength calibration of the instrument has not been done yet because the instrument is still undergoing changes to improve optical efficiency and stability. Over the next months these improvements will be completed and the final calibration will be performed. Additional data taken with this instrument will be available at the AMIGO website: http://acdb-ext.gsfc.nasa.gov/People/Weaver/AMIGO.html.

\section{CONCLUSIONS}

We are reporting a new improved filter radiometer which uses a CCD array to sample both the reference and the Fabry-Perot signal simultaneously on different areas of the array. It increases the signal to noise ratio (SNR) while permitting a reduction in the overall size of the instrument. It can evolve to become an extremely flexible instrument exploiting the superior throughput and resolution of the Fabry-Perot interferometer. With changes of the prefilter and the etalon the system can measure different greenhouse gases in the atmosphere.

\section{REFERENCES}

[1] http://www.xenics.com/en/xenics_infrared_solutions/xenics_infrared _solutions.asp

[2] B. Metz, O.R. Davidson, P.R. Bosch, R. Dave, L.A. Meyer , Contribution of Working Group III to the Fourth Assessment Report of the Intergovernmental Panel on Climate Change, 2007.

[3] Katey Walter Anthony, "Methane: A Menace Surfaces", Scientific American, December, 2009.

[4] U.S. Global Carbon Cycle Science Program 2007, Highlights of Recent Research and Plans for FY 2007, Chapter 5, pp.114-120.

[5] J. Lelieveld, P. J. Crutzen, and F. J. Dentener, "Changing concentration, lifetime and climate forcing of atmospheric methane," Tellus Series B-Chemical and Physical Meteorology, vol. 50, pp. 128-150, Apr 1998.

[6] Responding to the Challenge of Climate and Environmental Change: NASA's Plan for a Climate-Centric Architecture for Earth Observations and Applications from Space, June 2010.

[7] J. Vaughan, The Fabry-Perot Interferometer: history, theory, practice, and applications, Bristol, England, 1989.

[8] G. Hernandez, Fabry-Perot interferometers, Cambridge, New York, 1986.

[9] James W. Beletic et al., Teledyne Imaging Sensors: Infrared imaging technologies for Astronomy \& Civil Space, Proceedings of the SPIE Conference on Astronomical Instrumentation (2008, Marseille, France)

[10] Georgieva E.M., Wilson E.L., Miodek M., Heaps W.S., "Total Column Oxygen Detection Using Fabry-Perot Interferometer", Optical Engineering 45 (11), Paper No115001, 1- 11(2006).

[11] E.L. Wilson, E. M. Georgieva, W. S. Heaps, "Development of a Fabry-Perot interferometer for ultra-precise measurements of column CO2”, Measurement Science and Technology 18, 1495-1502 (2007).

[12] W.S. Heaps, E.L. Wilson, Georgieva E.M, Precision Measurement of Atmospheric Trace Constituents Using a Compact Fabry-Perot Radiometer", International Journal of High Speed Electronics and Systems (IJHSES), 18, 3, September, 2008.

[13] Georgieva E.M., W. S. Heaps, E. L. Wilson.: "Differential Radiometers using Fabry- Perot Interferometric Technique for Remote Sensing of Greenhouse Gases”, IEEE Transactions on Geoscience and Remote Sensing (TGARS), 46,10, October special issue, 2008.

[14] W.S.Heaps, "Broadband lidar technique for precision CO2 measurement", Proc. SPIE, Vol. 7111, 711102 (2008).

[15] D. T. Shindell, G. Faluvegi, N. Bell, and G. A. Schmidt, "An emissions-based view of climate forcing by methane and tropospheric ozone," Geophysical Research Letters, vol. 32, p. L04803, Feb 18 2005.

[16] C. Frankenberg, J. F. Meirink, M. van Weele, U. Platt, and T. Wagner, "Assessing methane emissions from global space-borne observations," Science, vol. 308, pp. 1010-1014, May 132005.

[17] Hansen, J. E., M. Sato, and R. Ruedy, "Radiative forcing and climate response”, J. Geophys. Res., 102, 6831- 6864, 1997.

[18] Hansen, J., M. Sato, R. Ruedy, A. Lacis, and V. Oinas, "Global warming in the twenty-first century: An alternative scenario", Proc. Natl. Acad. Sci. U.S.A., 97, 9875-9880, 2000.

[19] Jain, A. K., B. P. Briegleb, K. Minschwaner, and D. J. Wuebbles, "Radiative forcing and global warming potentials of 39 greenhouse gases”, J. Geophys. Res., 105, 20,773- 20,790, 2000.

[20] Kei Shiomi, Mayumi Yoshida and Nami Sekio, "Study for Sun Glint Observation of GOSAT FTS using MODIS and AMSR-E Data", IEEE, pp.2060-2063, 2006. 